\title{
Natural History and Predictors of recovery in Children with Chronic Spontaneous Urticaria
}

Luisa Cortellazzo Wiel ${ }^{1}$, Ester Conversano ${ }^{1}$, Manuela Giangreco ${ }^{2}$, Laura Fagotto ${ }^{1}$, Maria Rita Lucia Genovese $^{1}$, Laura Badina ${ }^{2}$, Giorgio Longo ${ }^{2}$, Egidio Barbi ${ }^{1,2}$, Irene Berti²

\section{Affiliations:}

${ }^{1}$ University of Trieste, Italy

${ }^{2}$ Institute for Maternal and Child Health - IRCCS "Burlo Garofolo" - Trieste, Italy

Ester Conversano, MD

University of Trieste, Department of Medicine, Surgery and Health Sciences

Via dell'Istria 65/1, 34137, Trieste, Italy

Tel: 00393335838780, E-mail: esterconversano@gmail.com, Fax: 00390403785290

\section{Natural History Chronic Spontaneous Urticaria.}

Word count: 1215

Tables: 2

Figures: 1

This article has been accepted for publication and undergone full peer review but has not been through the copyediting, typesetting, pagination and proofreading process, which may lead to differences between this version and the Version of Record. Please cite this article as doi: $\underline{10.1111 / \text { PAI.13299 }}$

This article is protected by copyright. All rights reserved 
DR ESTER CONVERSANO (Orcid ID : 0000-0003-4317-8231)

Article type : Letter to the Editor

\title{
Natural History and Predictors of recovery in Children with Chronic Spontaneous Urticaria
}

Conflict of interest: The authors declare that they have no conflict of interests.

Financial support: The authors declare that they had no financial support.

\begin{abstract}
:
Background: Limited data are available about the natural history of chronic spontaneous urticaria in children (CSU), and management guidelines are mostly extrapolated from evidence in adults.
\end{abstract}

Methods: The objective of this study was toevaluate the natural history of CSU in children and to identify predictors for remission. We performed an observational study, including patients aged 0 to 18 years with CSU diagnosed from January 2006 to July 2016. Disease activity was evaluated by the Urticaria Activity Score (UAS) and the Urticaria Activity Score 7 (UAS7); type of treatment and number of daily administrations were recorded.

Results: Eighty patients were included in the study. At 1, 3, and 5 years from the onset of symptoms, $29 \%, 55 \%$, and $72 \%$ of the patients with CSU were in remission, respectively. A higher hazard ratio of non-remission both at 3 years $(\mathrm{HR}=1.7,95 \% \mathrm{CI}=1.2-2.4$, p-value 0.004$)$ and at 5 years $(\mathrm{HR}=1.7$, $95 \% \mathrm{CI}=1.2-2.7$, p-value 0.001 ), was related to higher severity of CSU at the baseline. Remission rates were significantly higher in patients who used a single dose of antihistamine drug (89\% vs. $54 \%$ and $67 \%$ at 5 years, $p$-value $=0.0003)$. During follow-up, four patients experienced a relapse $(6.9 \%)$. The distance of relapses from remission varied from 2 to 5 years without evidence of a correlation between the age at the onset and the duration of the disease (Spearman's coefficient $=0.02$; pvalue $=0.9)$.

Conclusion: High UAS score at onset is a prognostic factor for the persistence of symptoms, as well as the need for multiple antihistamine drug doses per day or second-line treatments.

Keywords: chronic spontaneous urticaria; children; natural course; remission; UAS; UAS7; treatment; autologous serum skin test; autoimmunity; predictive factors.

This article is protected by copyright. All rights reserved 
To the Editor,

Chronic urticaria $(\mathrm{CU})$ is a relatively frequent disease with an estimated point prevalence of $0.7 \%$ around the world, subject to regional differences ${ }^{1}$. Children seem to display a slightly higher frequency of the disease compared to adults, but to date, limited epidemiological data are available regardingthe paediatric age, with only a few studies specifically examining the Caucasian population $^{1234}$.

Although it is considered a self-limited disease, CU often lasts for years. However, data on the natural historyof CU are lacking, particularly in children, and frequently are not specific to the different $\mathrm{CU}$ subtypes.Finally, management recommendations are mostly extrapolated from evidence in adults.

We aimed todescribethe natural history of Chronic Spontaneous Urticaria (CSU), the most frequent CU subtype, in a cohort of Caucasian children and to identify possible prognostic factors that could predict remission.

We performed an observational study at the Department of Paediatric Allergy and Immunology of the University teaching Tertiary Hospital, Institute for Maternal and Child Health IRCCS "BurloGarofolo" of Trieste. The Internal Review Board approved the study. Patients aged 0 to 18 years with CSU diagnosed from January 2006 to July 2016 were included. Inducible urticaria, disease onset more than two years before the first examination or coexisting underlying conditions such as cardiovascular, hepatobiliary, and renal diseases were considered as exclusion criteria. Medical records were evaluated retrospectively, and the patients were eventually contacted by telephone to investigate their current CSU status. Remission was defined as an urticaria free period of at least three months without treatment, while reappearance of symptoms after remission was considered as a relapse. Patients were divided into three groups to evaluate the effect of the treatment regimen on the remission rate. They were assigned to the first group if they used a single dose of antihistamine drug, to the second group if they needed more than one daily dose (two, three or four doses per day), and to the third group if they used an adjunctive therapy, such as cyclosporin A or montelukast. An Autologous Serum Skin Test (ASST) was offered and performed in a subpopulation of patients who agreed to be tested ${ }^{3}$. Disease activity was assessed at the onset and during every control visit by the Urticaria Activity Score (UAS) and the Urticaria Activity Score 7 (UAS7) ${ }^{3}$. The specific methods of data recording and statistical analyses are described in Appendix 1.

The primary outcome was to assess the natural history of CSU. The secondary outcome was to identify likely predictors of disease duration.

A total of 86 patients were considered eligible, 6 of whom were lost to follow-up. The final population examined consisted of 80 patients, 41 females $(51.3 \%)$, and 39 males (48.8\%). The demographic and laboratory characteristics of the enrolled patients are reported in Table 1. Cetirizine was the second-generation $\mathrm{H} 1$-antihistamine drug of choice for all patients, with an average dose of 
one per day (IQR: 1-2), being the sole treatment in 63 (78.8\%) cases; overall 36 children (45\%) took one dose per day of antihistamine and 27 (34\%) more than one. An adjunctive therapy was recorded in 17 children (21.3\%): all of them took corticosteroids, associated with another antihistamine in 2 cases $(2.5 \%)$, ciclosporin in 1 case $(1.3 \%)$ and montelukast in 1 case $(1.3 \%)$.

An ASST was performed in 24 children (30.0\%) with a positive result in 11 cases (45.8\%). The result of the ASST did not affect the remission rates (Table 2).

Six patients $(7.5 \%)$ presented elevated thyroid-stimulating hormone (TSH), despite normal free-T4 (fT4)with a median age at the finding of 9 years (IQR 4.75, 6.5-11.25), one patient (1.25\%) had positive anti-TPO detected since the age of one year. The onset of comorbidities after the diagnosis of CSU was recorded in two patients (2.5\%), consisting of celiac disease and hypothyroidism due to Hashimoto's thyroiditis, respectively, the latter presenting at the age of 16 years.

The median duration of CSU was 28 months (IQR: 9.0-50.0 months). At 1, 3 and 5 years of symptom onset, $29 \%, 55 \%$, and $72 \%$ of CSU patients were in remission, respectively, with a cumulative remission rate of $78 \%$.

Remission rate was significantly higher in the group of children who used a single dose of antihistamine drug per day than in the second and third group, respectively ( $81 \%$ vs. $30 \%$ and $44 \%$ at 3 years, p-value $<0.0001 ; 89 \%$ vs. $54 \%$ and $67 \%$ at 5 years, p-value $=0.0003$ ) (Figure $1 a$ and $1 b$ ).

The hazard ratio of the non-remission both at 3 years $(\mathrm{HR}=1.7,95 \% \mathrm{CI}=1.2-2.4$, $\mathrm{p}$-value 0.004$)$ and 5 years from the onset of disease $(\mathrm{HR}=1.7,95 \% \mathrm{CI}=1.2-2.7$, $\mathrm{p}$-value 0.001$)$, was higher for patients with a more severe UAS score at onset (Table 2).The median UAS score at onset was lower in the group of remission than in the group of non-remission both at 3 years (Median=2, IQR: $2-3$ vs. Median=5, IQR: 4 - 5) and 5 years from the onset of disease (Median=3, IQR: 2 - 4 vs. Median=5, IQR: 4 - 5).

There was no evidence of a correlation between age at the onset and duration of the disease (Spearman's coefficient $=0.02 ; \mathrm{p}$-value $=0.9$ ).

During the follow-up, four patients previously in disease remission had a relapse of CSU (6.9\%). The distance of relapses from remission ranged from 2 to 5 years, while the relapse was protracted from 4 to 12 months.

Several studies have focused on the identification of factors that can predict the natural course of the disease, as desirable in long course conditions such as $\mathrm{CSU}^{1-4}$.

We found a median duration of CSU of 28 months, consistent with that reported by Chansakulporn et al. of 33 months (2.7 years $)^{3}$.

We identified a remission rate at 1,3, and 5 years of 29\%,55\%, and 72\%, respectively, consistent with those found by Eser et al. (32.7\% and $46.1 \%$ at 1 and 3 years, respectively) ${ }^{4}$, although they differ from those reported by Sahiner ${ }^{2}$ and Chansakulporn ${ }^{3}$, which were both lower at one year. These data suggest a favorable trend of CSU in children, with the majority of patients reaching remission within five years. Although remission rates in adults are traditionally considered lower than in children, with 
$20 \%$ of patients with ongoing active disease at twenty years from onset ${ }^{5}$, according to recent studies, trends would be more consistent with those observed in the paediatric population ${ }^{6,7}$.

The duration of CSU might be predicted at the onset by the severity of the UAS score; moreover, patients needing more than one dose of antihistamine drugs per day displayed a lower remission rate at 3 and 5 years when compared to patients using standard doses (figure $1 a$ and $1 b$ ). These results are consistent with those reported by $\mathrm{Eser}^{4}$ and Yilmazet al. ${ }^{8,9}$. Rates of associated atopic disorders, family history of atopy, and possible autoimmune CSU etiology (as suggested by a positive ASST result) are similar to those reported in previous studies ${ }^{9}$. Nevertheless, none of these associated conditions seems able to impact on the remission rate. On the contrary,Netcvhiporouk et al. ${ }^{10}$ found a higher resolution rate in cases of paediatric onset CSU with biomarkers of autoimmunity,such as positive results of a basophil activation test (BAT) using CD63 activation marker. Possibly, BAT may be a more specific biomarker for autoimmunity than ASST, and further studies should clarify this issue.

A limitation of our study is the relatively small sample size and missing evaluation of treatmentcompliance. No patients in this cohort used omalizumab, as the study was conducted mostly before its recent approval for use in children aged 12 and over in Italy ${ }^{11}$.

In conclusion, this study showed that the prognosis of CSU is generally favourable in children, with a remission rate increasing up to $72 \%$ at 5 years after the onset of symptoms. Paediatric onset CSU is a benign entity, mostly easier to control by the first-line therapy than in adulthood, which instead requires second-line therapy in about two-thirds of the cases ${ }^{12}$. Disease severity (elevated UAS score at onset as well as the need for adjunctive treatments) seemed to predict a more long-lasting disease. Relapses can occur later after the first remission and are not related to the age at the onset or the duration of the disease.

Luisa Cortellazzo Wiel ${ }^{1}$, Ester Conversano ${ }^{1}$, Manuela Giangreco ${ }^{2}$, Laura Fagotto ${ }^{1}$, Maria Rita Lucia Genovese $^{1}$, Laura Badina ${ }^{2}$, Giorgio Longo ${ }^{2}$, Egidio Barbi ${ }^{1,2}$, Irene Berti $^{2}$

\author{
Affiliations: \\ ${ }^{1}$ University of Trieste, Trieste, Italy \\ ${ }^{2}$ Institute for Maternal and Child Health - IRCCS "BurloGarofolo", Trieste, Italy
}

Acknowledgements: The authors thanks Martina Bradaschia for the English revision of the manuscript.

\title{
References
}

This article is protected by copyright. All rights reserved 
${ }^{1}$ Fricke J, Avila G, Keller T, et al. Prevalence of chronic urticaria in children and adults across the globe: systematic review with meta-analysis. Allergy. 2020; 75: 423-32.

${ }^{2}$ Sahiner UM, Civelek E, Tuncer A et al. Chronic urticaria: etiology and natural course in children. International Archives of Allergy and Immunology 2011;156(2):224-30.

${ }^{3}$ Chansakulporn S, Pongpreuksa S, Sangacharoenkit P et al. The natural history of chronic urticaria in childhood: A prospective study. Journal of the American Academy of Dermatology 2014;71(4):6638.

${ }^{4}$ Eser I, Yologlu N, Baydemir C et al. The predictive factors for remission of chronic spontaneous urticaria in childhood: Outcome from a prospective study. Allergologia Et Immunopathologia2016;44(6):537-41.

${ }^{5}$ Greaves M. Chronic urticaria. J Allergy Clin Immunol. 2000;105:664-72.

${ }^{6}$ Curto-Barredo L, Archilla LR, Vives GR, Pujol RM, Giménez-Arnau AM. Clinical Features of Chronic Spontaneous Urticaria that Predict Disease Prognosis and Refractoriness to Standard Treatment. ActaDermVenereol. 2018 Jul 11;98:641-47.

7 Maurer M1, Weller K, Bindslev-Jensen C, et al. Unmet clinical needs in chronic spontaneous urticaria. A GALEN task force report. Allergy. 2011;66:317-30.

${ }^{8}$ ArikYilmaz E, Karaatmaca B, Cetinkaya PG et al. The persistence of chronic spontaneous urticaria in childhood is associated with the urticaria activity score. Allergy Asthma Proc. 2017;1;38(2):13642. 
${ }^{9}$ Cornillier H, Giraudeau B, Munck S. Chronic spontaneous urticaria in children - a systematic review on interventions and comorbidities. Pediatr Allergy Immunol. 2018;29:303-10.

${ }^{10}$ Netchipororuk E, Sasseville D, Moreau L, et al. Evaluating comorbidities, natural history, and predictors of early resolution in a cohort of children with chronic urticaria.JAMA Dermatol. 2017; 153(12)1236-42.

${ }^{11}$ Maurer M, Metz M, Brehler R et al. Omalizumab treatment in patients with chronic inducible urticaria: A systematic review of published evidence. J AllergyClinImmunol. 2018;141(2):638-49.

${ }^{12}$ Staevska M1, Popov TA, Kralimarkova T, et al. The effectiveness of levocetirizine and desloratadine in up to 4 times conventional doses in difficult-to-treat urticaria. J Allergy Clin Immunol. 2010;125:676-82. 
Table1: Demographical and laboratory characteristics of the study population at enrollment.

\begin{tabular}{|c|c|}
\hline & N. of patients (\%) Total 80 \\
\hline Females & $41(51.2 \%)$ \\
\hline Median age at onset & 7 \\
\hline$<5$ years & $24(30 \%)$ \\
\hline $5-10$ years & $36(45 \%)$ \\
\hline $11-15$ years & $19(23.8 \%)$ \\
\hline $16-18$ years & $1(1.2 \%)$ \\
\hline Median UAS score at onset & 3.5 (IQR: 2.0-5.0) \\
\hline Median UAS7 score at onset & 25.0 (IQR: $16.0-34.5)$ \\
\hline ASST (performed in 24 children, $30 \%$ ) & $11 / 24(45.8 \%)$ \\
\hline Personal history of atopy & $24(30.0 \%)$ \\
\hline - food allergies & $6(7.5 \%)$ \\
\hline - asthma & $2(2.5 \%)$ \\
\hline - allergic rhinitis & $16(20.0 \%)$ \\
\hline - atopic dermatitis & $10(12.5 \%)$ \\
\hline Family history of atopy & $33(41.3 \%)$ \\
\hline - food allergies & $2(2.5 \%)$ \\
\hline - asthma & $3(3.8 \%)$ \\
\hline - allergic rhinitis & $28(35.0 \%)$ \\
\hline
\end{tabular}

This article is protected by copyright. All rights reserved 


\begin{tabular}{|l|l|}
\hline \multicolumn{1}{|c|}{$\quad$ atopic dermatitis } & $4(5.0 \%)$ \\
\hline Elevated TSH with normal fT4 & $2(2.5 \%)$ \\
\hline Positive Anti-TPO titers & $6(7.5 \%)$ \\
\hline Hashimoto's thyroiditis & $1(1.3 \%)$ \\
\hline Positive Anti-TG titers & $1(1.3 \%)$ \\
\hline Positive ANA titers & $1(1.3 \%)$ \\
\hline IgA deficiency & $1(1.3 \%)$ \\
\hline
\end{tabular}

Table2. Cox proportional hazard models of non-remission at 3 and 5 years from the onset of disease

\begin{tabular}{|c|c|c|c|c|c|c|c|}
\hline \multirow[b]{2}{*}{ Independentvariables } & \multirow[b]{2}{*}{$\begin{array}{l}\text { Reference } \\
\text { category }\end{array}$} & \multicolumn{3}{|c|}{3 years } & \multicolumn{3}{|c|}{5 years } \\
\hline & & $\mathbf{H R}^{1}$ & $\mathrm{CI} 95 \%{ }^{2}$ & $\begin{array}{l}\text { P- } \\
\text { value }\end{array}$ & $\mathbf{H R}^{1}$ & CI95\% ${ }^{2}$ & $\begin{array}{l}\text { P- } \\
\text { value }\end{array}$ \\
\hline \multicolumn{8}{|l|}{ Personal atopy: } \\
\hline Yes & No & 1.3 & $0.6-2.7$ & 0.5 & 1.3 & $0.5-3.0$ & 0.6 \\
\hline \multicolumn{8}{|l|}{ Familialatopy: } \\
\hline Yes & No & 1.0 & $0.5-1.9$ & 0.9 & 1.1 & $0.5-2.4$ & 0.9 \\
\hline UAS Score at the onset & & 1.7 & $1.2-2.4$ & 0.004 & 1.7 & $1.2-2.7$ & 0.01 \\
\hline ASST Test: & & & & & & & \\
\hline Notcarried out & Negative & 1.2 & $0.5-2.8$ & 0.7 & 1.5 & $0.6-4.0$ & 0.4 \\
\hline Positive & & 1.0 & $0.4-2.8$ & 1.0 & 1.2 & $0.4-3.6$ & 0.8 \\
\hline \multicolumn{8}{|l|}{ Therapy: } \\
\hline $\begin{array}{l}\text { More than } 1 \text { daily dose } \\
\mathrm{AH}^{3}\end{array}$ & 1 daily dose of & 1.8 & $0.7-4.3$ & 0.2 & 1.2 & $0.5-3.3$ & 0.7 \\
\hline Adjunctivetherapy & $\mathrm{AH}^{3}$ & 1.2 & $0.4-3.3$ & 0.8 & 1.0 & $0.3-3.4$ & 1.0 \\
\hline
\end{tabular}

\section{${ }^{1} \mathrm{HR}=$ Hazard Ratio, ${ }^{2} \mathrm{CI} 95 \%=95 \%$ Confidence Interval, ${ }^{3} \mathrm{AH}=$ Anti-Histamine drug}

This article is protected by copyright. All rights reserved 


\section{Figure legend}

Figure 1a.Kaplan-Meier curves for the probability of remission at 3 years from the onset of Chronic Urticaria, stratified by therapy. The remission rate was $81 \%$ for children who used a single dose of antihistamine per day, vs. $30 \%$ for children who used more than one dose of antihistamine per day, and $44 \%$ for children who used adjunctive therapy, p-value $<0.0001$.

Figure 1b.Kaplan-Meier curves for the probability of remission at 5 years from the onset of Chronic Urticaria, stratified by therapy. The remission rate was $89 \%$ for children who used a single dose of antihistamine drug, vs. 54\% for children who used more than one dose of antihistamine drug, and $67 \%$ for children who used adjunctive therapy, $\mathrm{p}$-value $=0.0003$.

Note to figure $1 \mathrm{c}, 1 \mathrm{~d}$ : Therapy $1=$ one daily dose of anti-histamine drug; $2=$ More than one daily dose of anti-histamine drug; $3=$ Adjunctive therapy. 


\section{Appendix 1}

Methods

Patients' follow up clinical data were collected: personal or family history of atopic diseases (such as atopic dermatitis, asthma, allergic rhinitis), connective tissue diseases or auto-inflammatory syndromes, drug intake, presence of angioedema, signs of infection, triggers of urticaria (such as cold, exercise, hot shower) and recent travel history.

Laboratory investigations included full blood count, erythrocyte sedimentation rate (ESR), C-reactive protein (CRP), complement, total immunoglobulins E (IgE), total immunoglobulins A (IgA), antitransglutaminase antibody (tTG), antinuclear antibody (ANA), thyroid-stimulating hormone (TSH), free T4, anti-thyroid peroxisomal antibody (anti-TPO) and anti-thyroglobulin antibody (anti-TG) levels.

During their first evaluation, all children underwent a skin prick test for Dermatophagoidespteronyssinus, Dermatophagoidesfarinae, Alternaria, cat and dog epithelia, grass, tree, and weed pollens. Histamine (10 $\mathrm{mg} / \mathrm{ml}$ of histamine phosphate) was used as a positive control and $0.9 \%$ sterile saline as a negative control. The skin prick test result was considered positive if the wheal diameter was $3 \mathrm{~mm}$ or higher compared with the negative control. 
An Autologous Serum Skin Test (ASST) was offered and performed in a subpopulation of patients who agreed after patients and families grantedthe informed consent. It was executed using an intradermal skin test with $0.05 \mathrm{ml}$ of sterile, fresh autologous serum. Histamine $(10 \mu \mathrm{g} / \mathrm{ml})$ and $0.9 \%$ sterile saline were used as positive and negative controls, respectively. The result was considered positive when the wheal diameter was $1.5 \mathrm{~mm}$ or higher, compared with the reaction elicited by a control saline solution 30 minutes after the intradermal injection.

According to the allergology unit, follow up protocol, disease activity was evaluated at the onset, and during every control visit by the Urticaria Activity Score (UAS) and the Urticaria Activity Score 7 (UAS7). In essence, they consist of a daily record of wheals number (range 0-3) and pruritus intensity (range 0-3), rated for one day (range 0-6) and seven consecutive days (range 0-42) respectively.

Statistical analysis:

Continuous variables were described as median and interquartile range while categorical variables were synthesized as frequency and percentage.

Spearman's test was used to establish the correlation between two continuous variables as the distributions were non-gaussian.

The Kaplan-Meier survival analysis was computed to evaluate the probability of remission at 1, 3 and 5 years from the onset of CSU, both in the entire population and in the treatment groups.

Patients were divided into three groups to evaluate the effect of the administered therapies on the remission rate: they were assigned to the first group if they used a single dose of an antihistamine drug, to the second group if they used more than one daily dose (2, 3 or 4 doses/day) and to the third group if they used an adjunctive therapy. The Log-rank test was used to determine the difference in the survival curves.

The proportional hazard Cox model was constructed to assess the relationship between the nonremission rate and independent variables as the personal history of atopy, family history of atopy, UAS score at the onset, ASST test and therapy. P-value $<0.05$ was considered statistically significant. All analyses were performed using SAS software, Version 9.4 (SAS Institute Inc., Cary, NC, USA). 


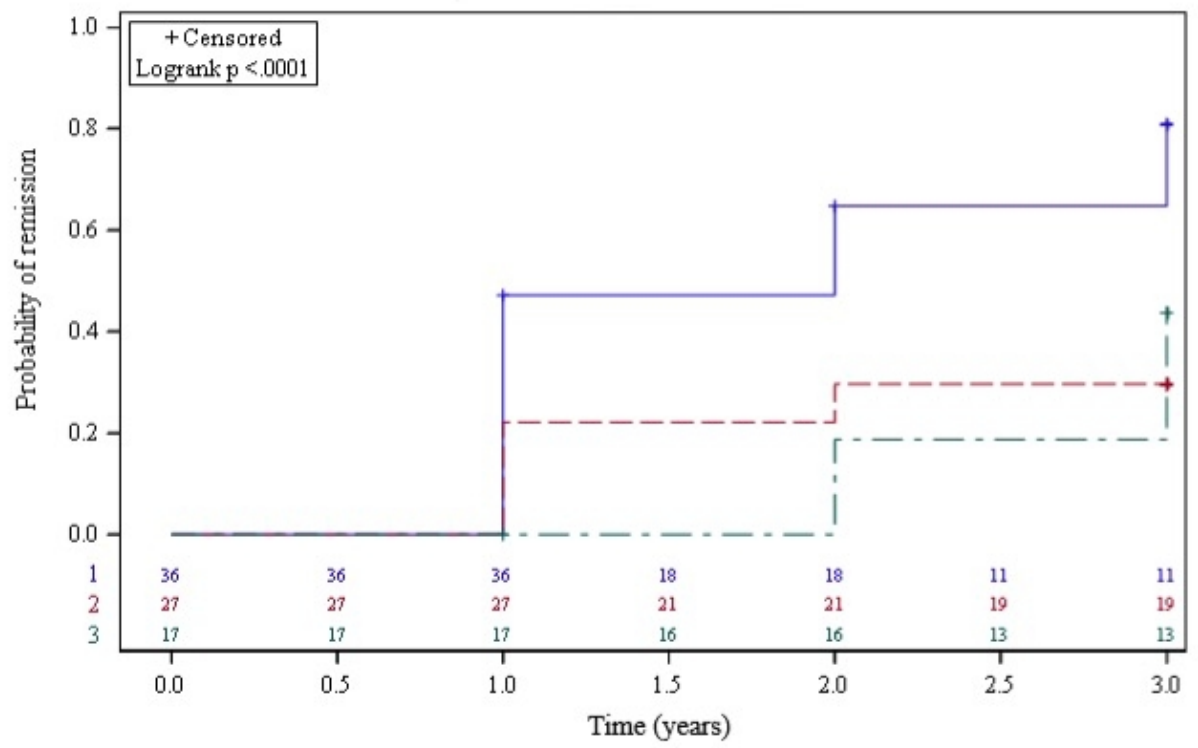

Therapy $-1---2-\cdots-3$

1a

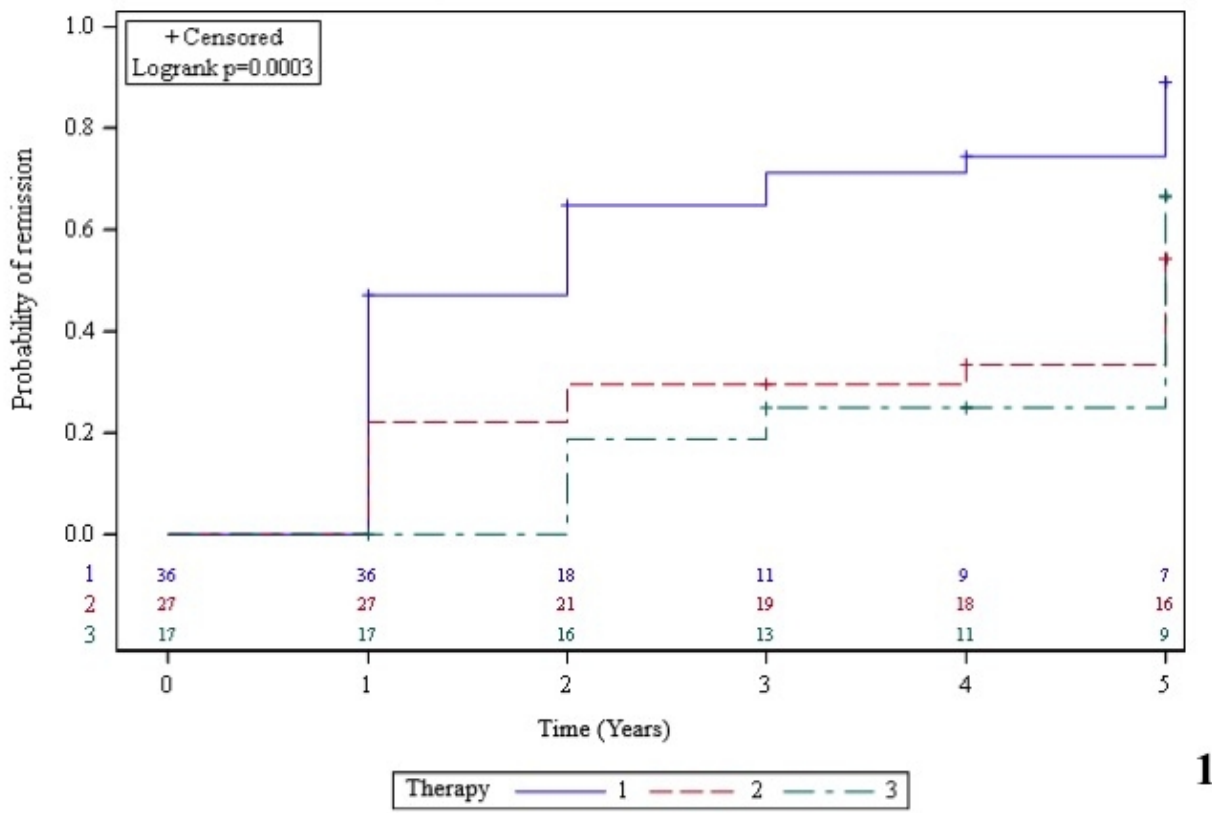

pai_13299_f1.jpg 